\title{
Experimental dynamic response of a nonlinear wire rope isolator
}

\author{
Andrea Salvatore ${ }^{1}$, Biagio Carboni ${ }^{1}$, Li-Qun Chen $^{2}$, Walter Lacarbonara ${ }^{1}$ \\ ${ }^{1}$ Department of Structural and Geotechnical Engineering, Sapienza University of Rome, Rome, \\ 00184, Italy \\ ${ }^{2}$ Shanghai Institute of Applied Mathematics and Mechanics, Shanghai University, Shanghai \\ 200072, China
}

\begin{abstract}
The steady-state dynamic response of a structure isolated by a nonlinear wire rope spring operating in the direction of gravity is experimentally studied. The isolated structure consists of two cantilever beams with a lumped mass at the tip. The forcedisplacement cycles provided by the isolator show a hysteretic behavior due to interwire friction and geometric nonlinearities. The restoring force is nonsymmetric exhibiting softening under compression and hardening under tension. The device rheological response is identified using experimental data and a suitable mechanical model. The frequency response curves (FRCs) for increasing levels of the vertical base excitation are obtained for the standalone device, the isolated and non-isolated structure. The expected softening trend of the isolation system as well as the increase of the displacement amplitude at low frequencies are ascertained both theoretically and experimentally. The comparison between the FRCs of the isolated and the non-isolated structure shows a severe reduction of the transmissibility coefficient in a broad frequency range. This work represents a first step towards the full modeling, validation of the reduced order model of the hysteretic isolator and the isolated structure towards a full optimization of the device isolation performance.
\end{abstract}

Keywords: Wire rope isolator, transmissibility, hysteresis, vibration isolation, system identification

\section{Introduction}

Wire ropes are commonly employed to sustain large axial loads. At the same time, they can be successfully exploited for applications in the field of structural vibration control. The first patented device based on steel wire ropes dates to the beginning of the last century and its scope consisted in the mitigation of galloping oscillations in cable electric transmission lines [1]. In recent years, several devices based on the hysteresis provided by wire rope assemblies have been proposed [2-7].

In this work, a nonlinear isolation system for reducing vertical vibrations is identified and experimentally investigated. The isolator is a so-called wire rope spring while the structure to be controlled is represented by two cantilevers with a tip mass undergoing bending motion. The nonlinear hysteretic behavior of the isolator is first characterized 
evaluating the equivalent stiffness and hysteretic damping at various displacement amplitudes and then identified through a suitable nonsymmetric hysteresis model. Subsequently, the test and the structure to be controlled are designed. Experimental FRCs of the isolated and non-isolated structure are finally obtained in order to estimate the transmissibility in the frequency bandwidth of interest.

\section{The isolator response}

The isolation system consists of a compact wire rope isolator (see Fig. 1). It is made of two metal plates joined by a stainless-steel wire rope having a diameter of $4 \mathrm{~mm}(7 \mathrm{x} 7$ wire of $0.4 \mathrm{~mm}$ ) [8]. The geometric nonlinearities of the cables induce an asymmetric hysteretic behavior. Hardening or softening is manifested under tension or compression, respectively. The device high deformability together with its high damping capacity makes it ideal for vibration control of machinery and other kinds of structures. Antonelli et al. investigated the hysteretic behavior of the device via quasi-static testing [Fig. 2] applying cyclic time histories with increasing amplitudes, namely $(0, \pm 2.5$, $\pm 5, \pm 7.5, \pm 10, \pm 12.5, \pm 15) \mathrm{mm}$.

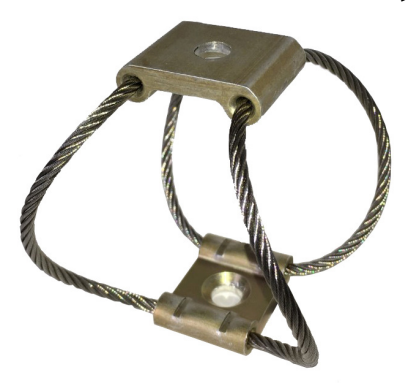

Fig. 1. The compact wire rope isolator

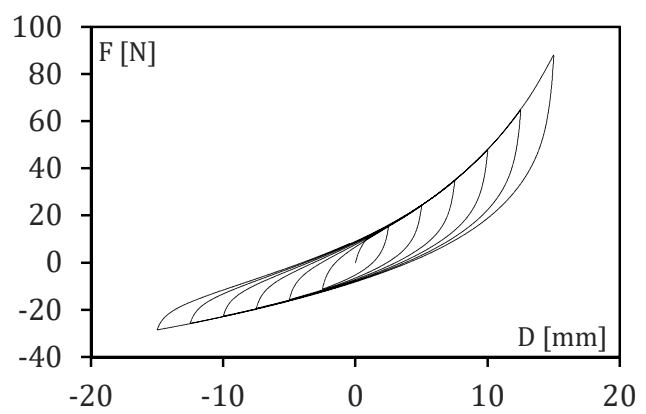

Fig. 2. Hysteretic loops under cyclic tests

The equivalent stiffness and damping versus displacement are obtained at each quasistatic cyclic amplitude proving the softening behavior under compression and the softening-hardening behavior under tension with a decreasing trend in equivalent hysteretic damping (Fig. 3).
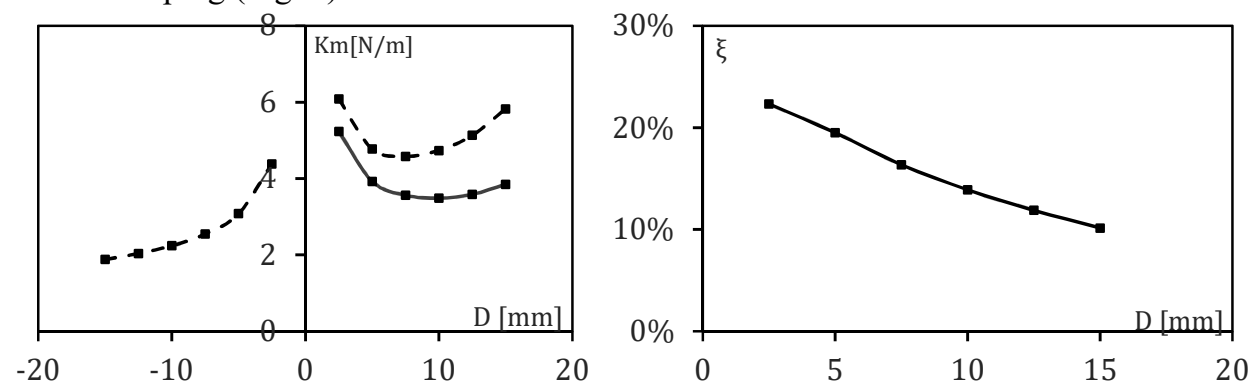

Fig. 3. (left) Stiffness vs. displacement (Tension and compression stiffness represented by dotted line, the average stiffness is indicated by solid line) and (right) equivalent hysteretic damping vs. displacement 
Starting from the modified asymmetric Bouc-Wen model of hysteresis proposed by Carboni et al. [9], a further generalization of the asymmetric constitutive response is proposed and here implemented. By employing the differential evolution algorithm [10], the model parameters which best fit the experimental quasi-static force-displacement cycles at different amplitudes are found (see Fig. 4).
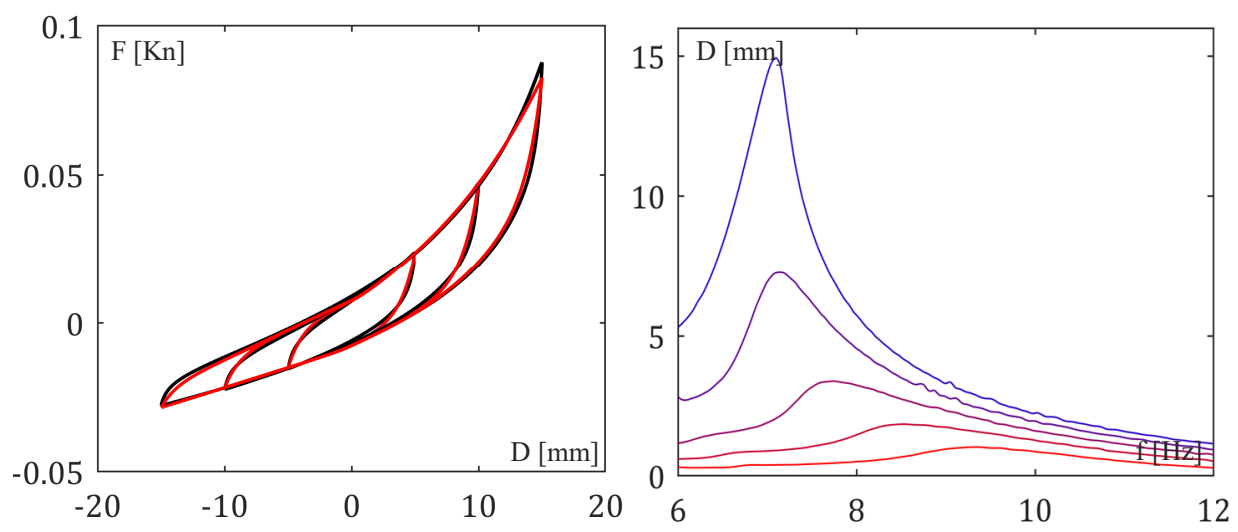

Fig. 4. (left) Numerical (red) vs. experimental (black) force-displacement cycles and (right) numerically obtained FRCs for the device with a mass of $1 \mathrm{~kg}$ for different base accelerations (i.e., 0.25 , red, $0.5,0.75,1.0,1.25 \mathrm{~g}$, blue)

\section{Experimental setup}

The structure to control during the test was designed starting with the stiffness of the device in order to obtain a frequency shift, between the non-isolated and the isolated structure, of about five times. The designed structure consists of two symmetric cantilever steel beams $(b=30 \mathrm{~mm}, \mathrm{~h}=2 \mathrm{~mm}, \mathrm{l}=7.5 \mathrm{~cm})$ with a tip mass of $0.24 \mathrm{~kg}$ for both beams. In order to evaluate the FRCs and the transmissibility over the investigated frequency range, for both the non-isolated and the isolated systems, shaker tests were carried out in frequency sweeps (i.e., sinusoidal input with constant amplitude and varying frequency).
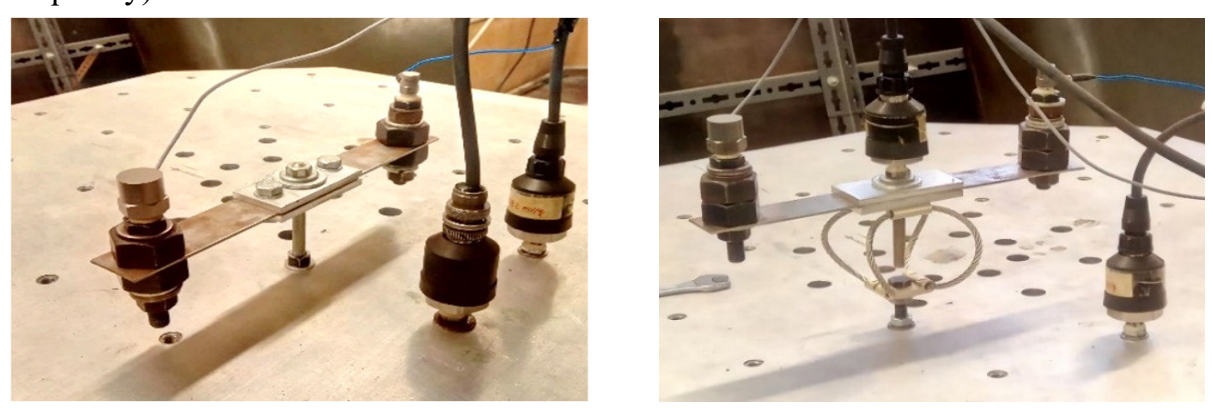

Fig. 5. Test setup for (left) the non-isolated structure and (right) the isolated structure 
The range of investigated frequencies was between $5 \mathrm{~Hz}$ and $100 \mathrm{~Hz}$ with a logarithmic sweep of $0.5 \mathrm{oct} / \mathrm{min}$. The test was repeated four times for both systems with different acceleration amplitudes. The accelerations of the masses $(0.24 \mathrm{~kg} \times 2)$ at the beam tips, of the mass mounted on the support $(0.45 \mathrm{~kg})$ and of the shaker head were acquired through four PCB Piezotronic accelerometers.

\section{Dynamic behavior of the uncontrolled structure}

For the considered range of base motions, the non-isolated structure responds linearly, so the theoretical FRC curves can be obtained with acceptable approximation in closed form. The steady-state response in displacement, $x_{s s}(\Omega)$, and acceleration, $\ddot{x}_{s s}(\Omega)$, and the linear transfer function $\mathrm{H}(\Omega)$ are given by: $x_{s s}(\Omega)=\mathrm{H}(\Omega) \ddot{x}_{g}, \ddot{x}_{s s}(\Omega)=$ $\Omega^{2} \mathrm{H}(\Omega) \ddot{x}_{g}, \mathrm{H}(\Omega)=(\mathrm{m} / \mathrm{k}) / \sqrt{\left[1-\left(\Omega / \omega_{n}\right)^{2}\right]^{2}+\left(2 \xi \Omega / \omega_{n}\right)^{2}}$, where $\Omega$ is the excitation frequency and $\omega_{n}$ the frequency of the $n$th mode of the structure. The structural stiffness is that of a cantilever beam with a point load at the tip. By knowing the mass, the governing parameters are the elastic constant and the damping ratio; namely, $k=$ $a \cdot E I / l^{3}$ where the constant $a=3$ for a perfect clamp; $\xi=c /\left(2 m \omega_{n}\right)$ is the damping ratio expressed in terms of the viscous damping coefficient $c$ which embodies the beam material damping and the boundary friction.

The frequency sweep tests were repeated for four different amplitudes and for each amplitude the stiffness and damping constant which better fit the experimental FRCs were found (see Table 1). The accelerations registered by the accelerometers were first filtered through the Savinskty-Golay Filter [11] and then doubly integrated to obtain velocities and displacements. The results of the integration were filtered again by a high-pass filter to remove the integration drift. In the subsequent figures the experimental FRCs (Fig. 6) and the trend of the transmissibility function (Fig. 7) are reported.

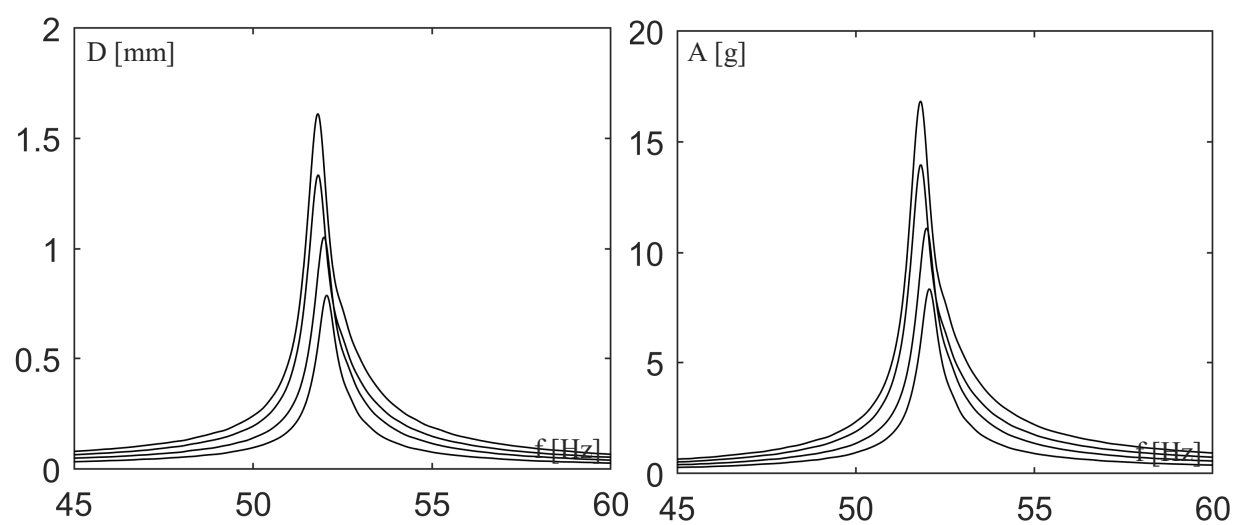

Fig. 6. Experimental FRCs in terms of (left) displacement and (right) acceleration for base accelerations of $(0.1,0.15,0.2,0.25) \mathrm{g}$ 


\begin{tabular}{|llll|}
\hline $\mathrm{N}_{\text {test }}$ & $\mathrm{Ag}[\mathrm{g}]$ & $a$ & $\xi[\%]$ \\
\hline 1 & 0.10 & 2.71 & $0.61 \%$ \\
2 & 0.15 & 2.70 & $0.66 \%$ \\
3 & 0.20 & 2.68 & $0.74 \%$ \\
4 & 0.25 & 2.68 & $0.74 \%$ \\
\hline
\end{tabular}

Table 1. Base accelerations, stiffness constant and damping ratio for each test

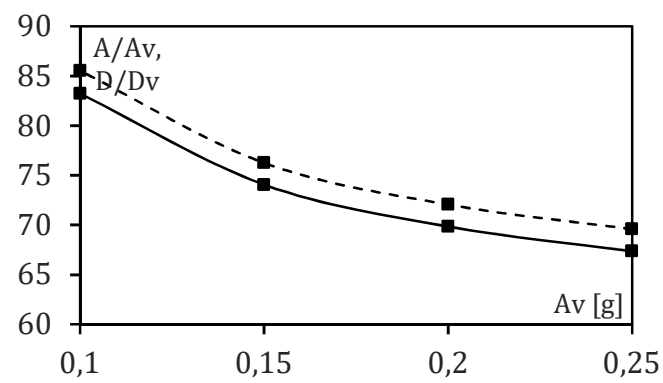

Fig. 7. Displacement (dotted) and acceleration (cont.) transmissibility peak vs. accel. amplitude

\section{Experimental response of the isolated structure}

The same frequency sweep tests were repeated for four different excitation levels for the isolated structure. As in the previous tests, displacements of each mass can be obtained by double integration and filtering of the acquired accelerations. The following figures (Fig. 8) show the comparison between the experimental FRCs in displacement and acceleration for the isolated (red) and non-isolated structures (black) for an input acceleration of $0.25 \mathrm{~g}$.
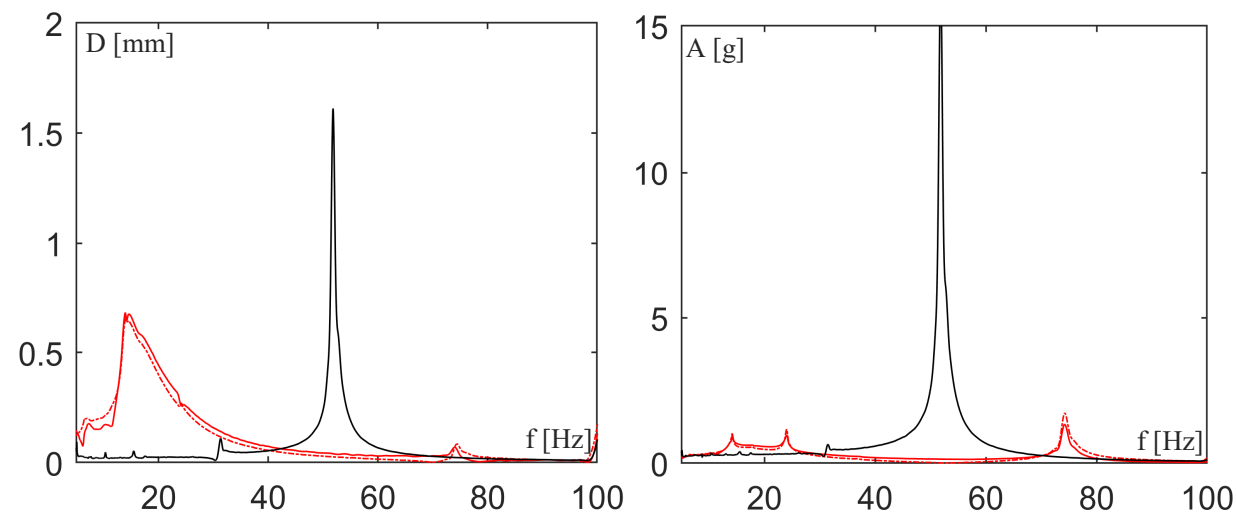

Fig. 8. Experimental FRCs of the uncontrolled structure (black line) vs. controlled structure (the red solid lines represent the response of the mass at the beam tip, while the red dashed lines indicate the response of the lumped mass on the isolator): (left) displacement and (right) acceleration

A strong reduction of the maximum acceleration of the isolated system can be observed due to the high compliance (hence, low frequency) of the device. As expected, at low frequencies the displacement of the isolated structure is larger than that of the nonisolated structure. At the same time, the maximum displacement of the isolated structure (elongation of the isolator) is indeed much smaller than the maximum displacement 
of the non-isolated structure (flexural beam vibration), thanks to the energy dissipation provided by the device built-in hysteresis. The subsequent figures (Fig. 9) represent the displacement and acceleration FRCs for the isolated structure for different excitation amplitudes (i.e., $0.25,0.5,0.75,1) \mathrm{g}$. The first and the second resonance frequencies, about $14 \mathrm{~Hz}$ and $24 \mathrm{~Hz}$, are relative at the elongation modes of the isolator. The third mode, near $74 \mathrm{~Hz}$, is the flexural mode of the beam. Furthermore, please note that the mass on the beam functions as a vibration absorber for the constraint mass at the frequency of $70 \mathrm{~Hz}$, making its relative acceleration vanish.
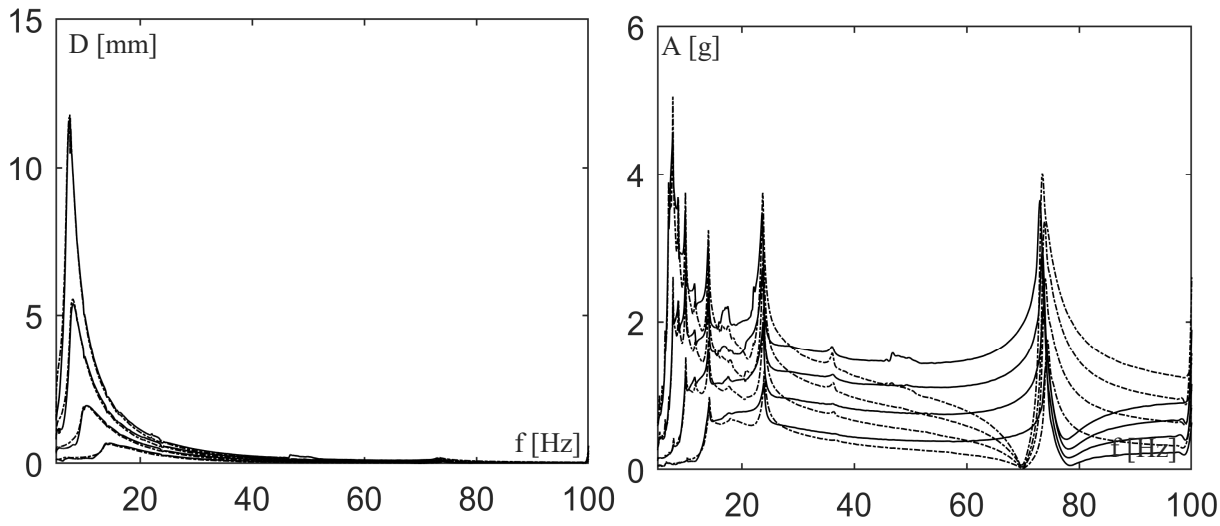

Fig. 9. (left) Displacement FRC and (right) acceleration FRC of the isolated structure for different excitation amplitudes (i.e., $0.25,0.5,0.75,1) \mathrm{g}$. The solid lines represent the response of the mass at the beam tip, while the black dashed lines indicate the response of the lumped mass on the isolator.

By focusing on the frequency range of the two last modes, we can observe a softening trend on both $(24 \mathrm{~Hz}$ and $74 \mathrm{~Hz})$ due to the softening geometric nonlinearities of the device (see Fig.10).
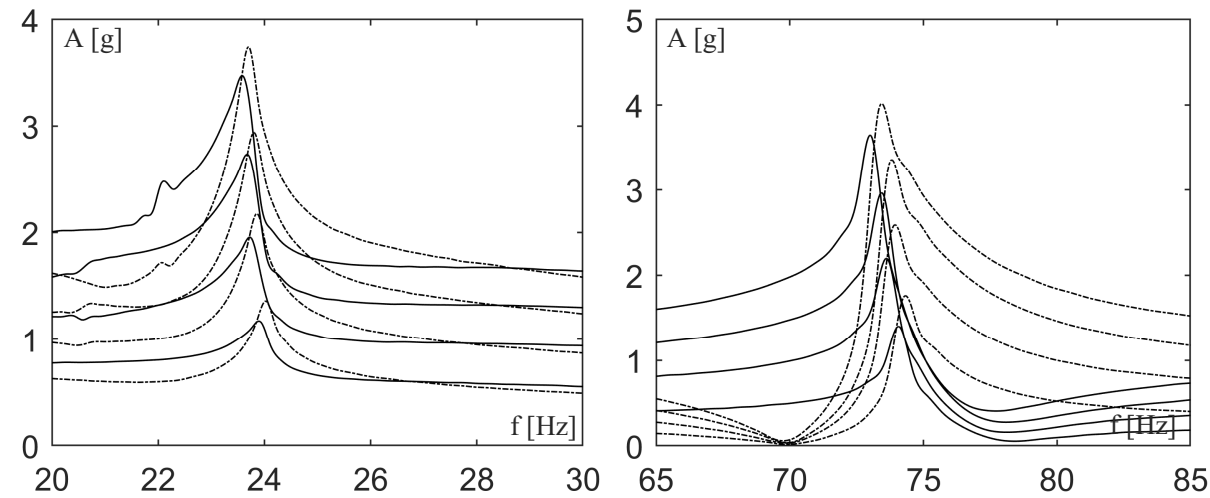

Fig. 10. Acceleration FRC near (left) the 2nd mode and (right) the 3rd mode of the isolated structure. Solid lines indicate the response of the mass on the beam and dotted lines denote the response of the mass on the isolator. 
The transmissibility is reduced by an order of magnitude over the entire frequency range examined (see Fig.11). In fact, it goes from an average value of about 75 for both the acceleration and displacement factors to an average value of about 6 . The transmissibility factor is evaluated for the third mode $(74 \mathrm{~Hz})$, which exhibits the maximum acceleration and the maximum displacement. The transmissibility factor shows a decreasing trend due to the softening behaviour which causes a more pronounced decoupling between the structure and the ground for larger excitation amplitudes (see Fig. 12 left). By making use of considerations of balance of linear momentum, the restoring force of the isolator during the test is also estimated. Fig. 13 right shows the forcedisplacement cycles at the resonance frequency of the isolator for four amplitudes. The agreement with the hysteretic loops provided by the quasi-static cyclic tests confirms the hardening-softening asymmetric behavior. There is also a drift because of the system weight during the dynamic tests, differently from the static tests.
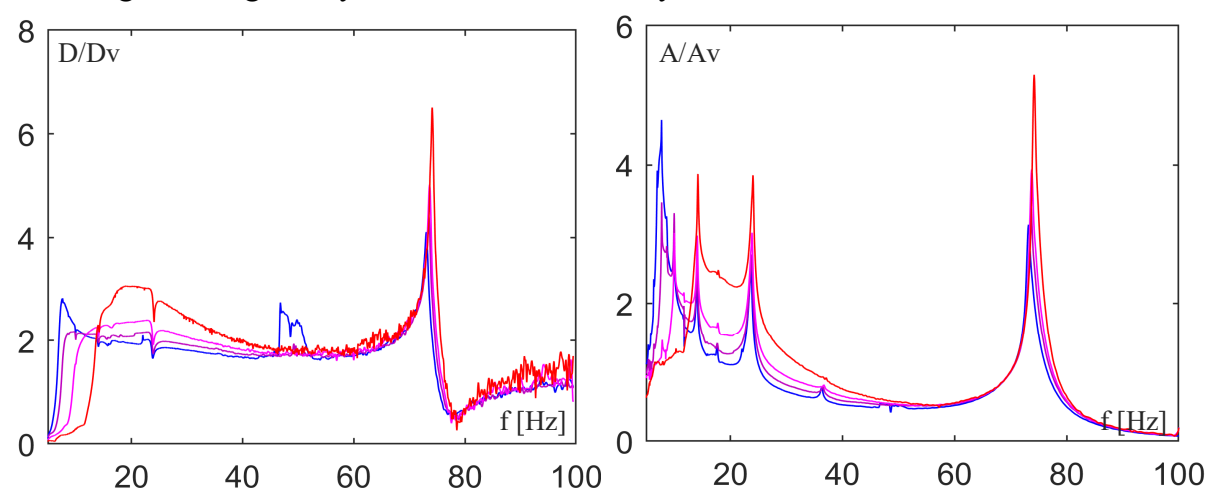

Fig. 11. (left) Displacement transmissibility and (right) acceleration transmissibility vs. frequency of isolated structure for an excitation amplitude of 0.25 (red), 0.5, 0.75, $1.0 \mathrm{~g}$ (blue).
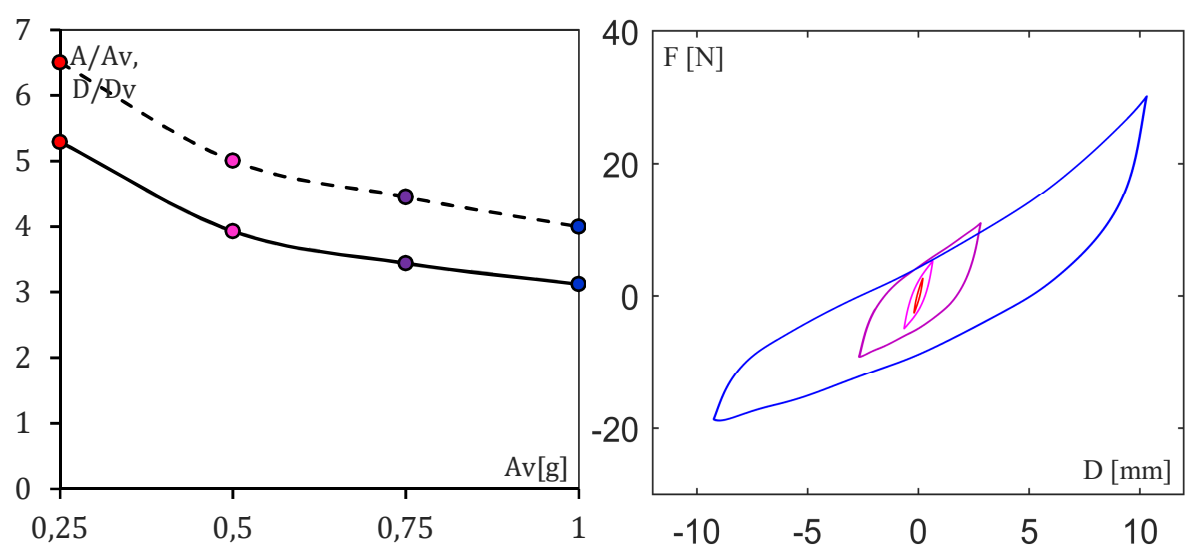

Fig. 12. (left) Displacement (dotted line) and acceleration (solid line) transmissibility vs. acceleration amplitude and (right) force-displacement cycle of the device for the four test amplitudes. 


\section{Conclusions}

The performance of the investigated hysteretic, nonsymmetric wire rope isolator shows a strong reduction of the transmissibility both in terms of acceleration and displacement over a wide frequency range. The hysteretic restoring force provided by the wire ropes under compressional and tensile cyclic loads was identified and it is shown to be suitable for vibration damping applications because of the large dissipation rate. This can mitigate the well-known drawback of isolation systems arising from the large compliance at low frequencies. However, the main problem with this type of devices for large scale structural isolation is the relative size between the device and the structure to be controlled. The present paper sets the initial steps of a wider experimental and theoretical work aimed at validating a reduced order nonlinear model capable of reproducing accurately the dynamic behavior of the device and the structure in order to achieve a wide nonlinear isolation system optimization.

\section{References}

1. George H. Stockbridge. Vibration damper. US Patent 1675931, 3 July 1928, Los Angeles, Usa.

2. Tinker, M. L., and Cutchins, M. A. Damping phenomena in a wire rope vibration isolation system. J. Sound Vib., 157, 718. (1992).

3. Demetriades, G. F., Constantinou, M. C., and Reinhorn, A. M. Study of wire rope systems for seismic protection of equipment in buildings. Eng. Struct., 15, 321334 (1993).

4. Gerges, R. R., and Vickery, B. J. Parametric experimental study of wire rope spring tuned mass dampers. J. Wind. Eng. Ind. Aerodyn., 91, 13631385. (2003)

5. Gerges, R. R., and Vickery, B. J. Wind tunnel study of the across-wind response of a slender tower with a non-linear tuned mass damper. J. Wind. Eng. Ind. Aerodyn.,91, 10691092. (2003)

6. Carboni, B., Lacarbonara, W. A nonlinear vibration absorber with pinched hysteresis: theory and experiments. Journal of Engineering Mechanics 142(5),04016023, (2015).

7. Carboni, B., Lacarbonara, W. Nonlinear dynamic characterization of a new hysteretic device: experiments and computations. Nonlinear Dynamics 83.1-2: 23-39, (2016).

8. Compact Wire rope Isolator, ITT Enidine Inc., 7 Centre Drive Orchard Park, NY, Usa.

9. Carboni, B., Lacarbonara, W., Brewick, P. T., \& Masri, S. F. (2018). Dynamical response identification of a class of nonlinear hysteretic systems. Journal of Intelligent Material Systems and Structures, 1045389X18778792.

10. Storn, R., Price, K. (1997) Differential Evolution - A Simple and Efficient Heuristic for global Optimization over Continuous Spaces. Journal of Global Optimization, 11:341. https://doi.org/10.1023/A:1008202821328

11. A. Savitzky, M.J.E. Golay, Smoothing and differentiation of data by simplified least-squares procedures, Anal. Chem. 36 (8) (1964) 1627-1639. 